\title{
Note préliminaire sur l'épidémiologie de la distomatose bovine au Sénégal
}

\author{
par S. GRÉTILLAT
}

La distomatose bovine existe au Sénégal et les rapports annuels du Service de l'Elevage signalent, dans les relevés des abattoirs de Saint-Lovis, Dakar, Louga, Thiès, Dagana, Podor, Mbaké, Kaolack, Tambacounda, Kedougou, Vélingara, Sediou et $\angle$ iguinchor, l'existence de douves dans les canaux biliaires des bovins abattus (MOREL, 1959).

II va sans dire que le fait de trouver des douves dans le foie d'un bovin sacrifié à l'abattoir, n'implique pas obligatoirement l'existence de la distomatose bovine dans la région.

En effet, à part le cas bien particulier du petit abattoir placé au centre d'une zone d'élevage productrice d'animaux de boucherie $\left({ }^{*}\right)$, la transhumance, et surtout les transactions commerciales, ne permettent pas en général de connaître l'origine des bovins abattus ef les endroits où ils ont contracté leur parasitisme.

La répartition géographique d'une maladie parasitaire à Trématodes ne peut done être connue que par l'examen des conditions épidémiologiques présentées par chaque région, ef par la connaissance de l'hôte intermédiaire du parasite.

En ce qui concerne la distomatose bovine, c'est, au Sénégal, Fasciola gigantica Cojboid. 1885, (douve géante) qui est le parasite en cause.

Ce trématode a besoin, pour accomplir son: cycle évolutif, de la présence d'un gastéropode d'eau douce du genre Lymnaea, Lamarch, 1779.

Nous avons pensé qu'en examinant le régime hydraulique et la faune malacologique des points d'eau de différentes régions du Sénégal, il nous serait peut-être possible de déterminer

(*) Celui de Kolda, par exemple.

Rev. Elev. Méd. vét. Pays trop., 1961, 14, n० 3.

Reçu pour publication : juin 1961.
I'hôte intermédiaire vecteur de Fosciola gigantica dans rette partie de l'Ouest-Africain.

Des enquêtes malaco-épidémiologiques faites au cours de l'année 1960 en Haute-Casamance, dans le Sine-Saloum, au Sénégal oriental, dans le Cayor, dans la presqu'île du Cap Vert, et des essais d'infestation expérimentale sur des mollusqiues d'élevage nous ont permis de déterminer:

to L'hôte intermédiaire de F. gigantica au Sénégal :

$2^{\circ}$ Certains facteurs écologiques et épidémiologiques expliquant la fréquence ou la rareté de la distomatose bovine dans des régions où existe pourtant son mollusque vecteur.

\section{ENQUETTE MALACO-ÉPIDÉMIOLOGIQUE FAITE EN HAUTE-CASAMANCE}

Le fleuve Casamance dans sa partie amont a un débit extrêmement variable suivant les époques de l'année.

A. Kolda, à environ 30 kilomètres de sa source (fig. 1), c'est, en fin de saison sèche (avril-mai), une petite rivière de quelques mètres de large sur 30 à $50 \mathrm{~cm}$ de profondeur. Par contre, pendant toute la saison des pluies (juin à novembre), c'est un véritable fleuve de plusieurs dizaines de mètres de largeur.

A.u cours d'une enquête malaco-épidémiologique effectuée vers la fin de juin 1960, nous avons trouvé, dans la Casamance et ses affluents (marigots), une abondante faune malacologique représertée par les espèces suivantes:

Biomphalaria pfeifferi goudi Ranson.

Bulinus guernei Dautzemberg.

1 Bulinus senegalensis Müller. (Groupe forskalii).

Lymnoe calliaudi Bourguignat.

Anisus sp. 


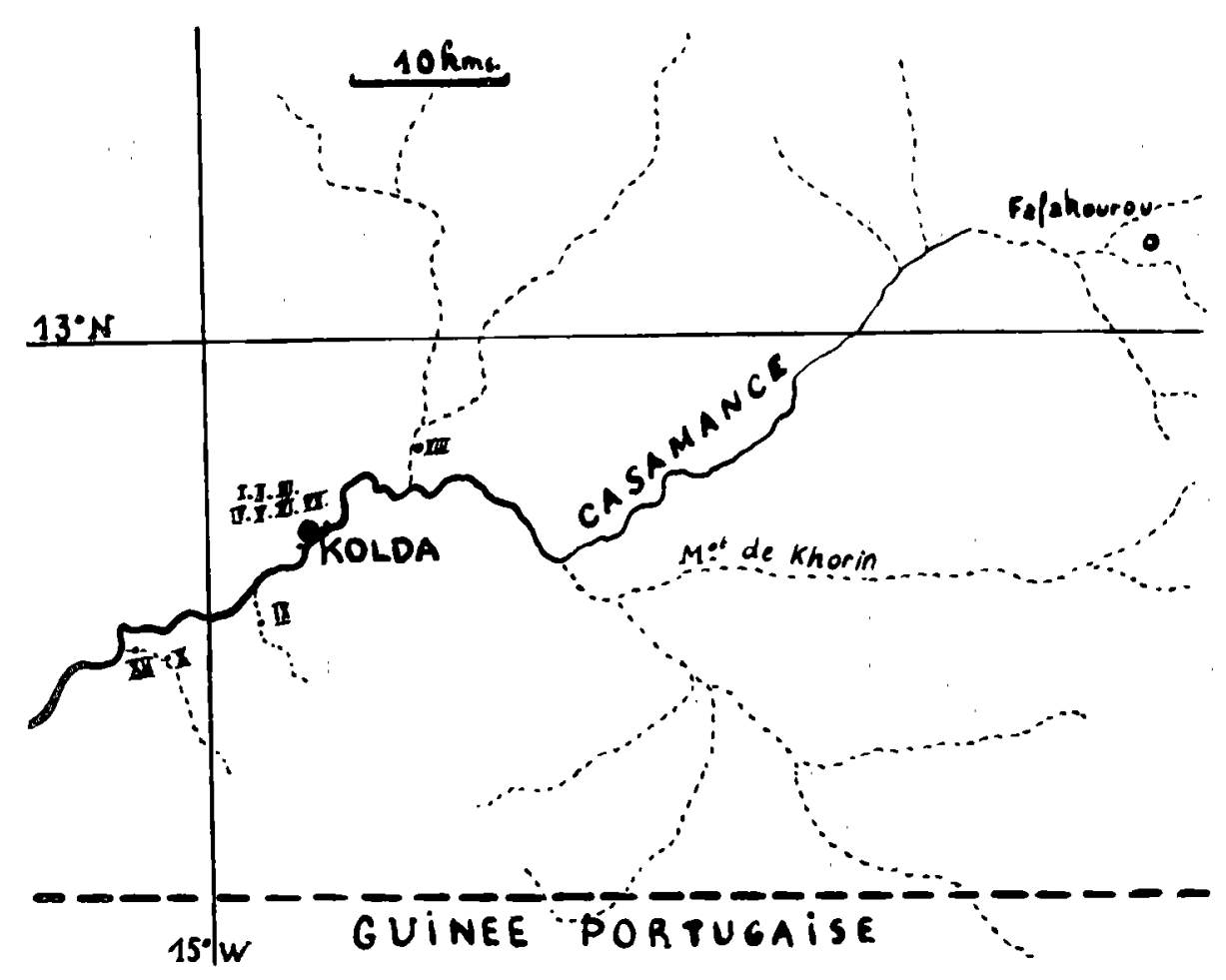

Fig. 1. - Carte de la région de Kolda.

En pointillé, les marigots affluents de la Casamance.

Les chiffres romains indiquent l'emplacement ef le numéro de chaque gîte à mollusques prospecté se rapportant au tableau no 1.

Le tableau no 1 résume les résultats de cetfe enquête malacologique et donne les principales conditions présentées par les différents gîtes : nature du fond et de la flore aquatique, température et $\mathrm{pH}$ de l'eau, nature du support où ont été trouvés les mollusques.

Les signes $(t)$ indiquent pour chaque espèce récoltée, sa densité approximative par mètre carré de surface de gîte.

$$
\begin{aligned}
+ & =2 \text { à } 3 \text { par mètre carré } \\
++ & =3 \text { à } 5 \text { par mètre carré } \\
+++ & =5 \text { à } 10 \text { par mètre carré } \\
++++ & =\text { plus de } 10 \text { par mètre carré. }
\end{aligned}
$$

Pour l'évaluation du chiffre : nombre de mollusques $/ \mathrm{m}^{2}$ nous délimitons en les jalonnant, deux surfaces carrées de 5 mètres de côté, dans deux endroits choisis du gîte à prospecter. Le même opérateur récolte pendant 15 minutes, dans chacune des aires ainsi déterminées, tous les mollusques visibles en surface et en profondeur. Les récoltes une fois dénombrées sont divisées chacune par 25. Les deux résultats obtenus, et arrondis à un nombre entier, donnent le maximum et le minimum par unité de surface de gîte.

\section{Résultats trouvés à la dissection des mollusques récoltés.}

Les pourcentages d'infestation, ainsi que la nature des formes larvaires de trématodes trouvés à la dissection des mollusques récoltés sont donnés dans le tableau no II. (Une centaine de spécimens disséqués pour chaque espèce).

\section{FRÉQUENCE DE LA DISTOMATOSE BOVINE EN RÉGION DE KOLDA}

(Enquête faite aux abattoirs)

Pendant notre séjour à Kolda, du 29.6 .60 au 5.7.60, nous avons pu examiner à l'abattoir local les viscères de 24 bovins. 
TABLEAJ 1 - Résultats obtenus au cours des cnquêtes malacologiques effectuées ein région de Kolda

\begin{tabular}{|c|c|c|c|c|c|c|c|c|c|c|c|}
\hline \multirow{2}{*}{ Getes } & \multirow{2}{*}{ Nature } & \multirow{2}{*}{ Fonds } & \multirow{2}{*}{ Flore } & \multirow{2}{*}{0} & \multirow{2}{*}{$\mathrm{pH}$} & \multirow{2}{*}{ Support } & \multicolumn{5}{|c|}{ Espèces récoltées } \\
\hline & & & & & & & Biom。 & BuI. & Anis. & Lysa. & B.s. \\
\hline I Kolda & $\begin{array}{l}\text { Casamance } \\
\text { rive droite en } \\
\text { amont du pont }\end{array}$ & \pm vaseux & graminées & $28^{\circ}$ & 6,6 & bois pourri & rares & $+1+$ & + & H+ & + \\
\hline II Kolda & $\begin{array}{r}\text { Casamance } \\
\text { sous le pont }\end{array}$ & pierreur & néant & $27^{\circ}$ & $6, \varepsilon$ & $\underset{\text { pont }}{\text { ciment du }}$ & & H+ & + & +4 & + \\
\hline III Kolda & $\begin{array}{l}\text { Casamence } \\
\text { milieu du fleuve } \\
\text { en amont du pont } \\
\text { à } 300 \mathrm{~m}\end{array}$ & vaseux & $\begin{array}{l}\text { némuphars } \\
\text { Pistia } \\
\text { Aeshornia }\end{array}$ & $28^{\circ}$ & 6,7 & nénuphars & + & H & + & + & +t \\
\hline IV Kolda & \begin{tabular}{l}
\multicolumn{1}{c}{ Casamance } \\
milieu du fleuve \\
en amont du pont \\
à 100 il
\end{tabular} & vaseux & $\begin{array}{l}\text { nénuphars } \\
\text { Pistia } \\
\text { Aeshornia }\end{array}$ & $30^{\circ}$ & 6,7 & $\begin{array}{l}\text { nénuphars } \\
\text { Pistia }\end{array}$ & + & $H$ & + & $\begin{array}{l}++ \\
+\end{array}$ & +++ \\
\hline $\begin{array}{ll}v & \\
& \text { Kolda }\end{array}$ & $\begin{array}{l}\text { Casanance } \\
\text { au niveau déver- } \\
\text { soir WC. publics }\end{array}$ & vaseux & $\begin{array}{l}\text { némuphars } \\
\text { Pistia }\end{array}$ & $30^{\circ}$ & 6,8 & $\begin{array}{l}\text { némughars } \\
\text { Pistia }\end{array}$ & & ++ & + & + & + \\
\hline${ }^{\text {VI }}$ Kolda & $\begin{array}{l}\text { Casamance } \\
\text { rive gauche en } \\
\text { amont du pont }\end{array}$ & \pm vaseux & graminées & $27^{\circ}$ & & bois pourri & & ++ & & + & $H$ \\
\hline VII Yolda & $\begin{array}{l}\text { Casamance } \\
\text { milieu du fleuve } \\
\text { en face abattoir }\end{array}$ & vaseux & $\begin{array}{l}\text { némuphars } \\
\text { Pistia } \\
\text { Aeshorria }\end{array}$ & $28^{\circ}$ & 6,8 & nénuphars & & & + & & + \\
\hline $\begin{array}{l}\text { VIII } \\
\text { Kolda }\end{array}$ & $\begin{array}{l}\text { Marigot en bor- } \\
\text { dure foret clas- } \\
\text { sée de Bahor - } \\
\text { route de Tamba- } \\
\text { counda }\end{array}$ & $\begin{array}{l}\text { maxigot } \\
\text { en crue }\end{array}$ & & $27^{\circ}$ & & $\begin{array}{l}\text { Pistia à la } \\
\text { dérive ou } \\
\text { bois pourri }\end{array}$ & & & & + & + \\
\hline $\begin{array}{l}\text { IX } \\
\text { Sarekeita } \\
6 \mathrm{~km} \text { Kolda }\end{array}$ & $\begin{array}{l}\text { Slarigot en } \\
\text { crue }\end{array}$ & vaseux & Pirtia & $28^{\circ}$ & 5,8 & $\begin{array}{l}\text { Pistia } \\
\text { bois poumri } \\
\text { Eraninées }\end{array}$ & & + & & + & ++ \\
\hline $\begin{array}{l}\mathrm{X} \\
\text { Britankountou } \\
14 \mathrm{~km} \text { iolda }\end{array}$ & Tete de zarigot & vaseux & $\begin{array}{l}\text { Ezstia } \\
\text { némupiass }\end{array}$ & $27^{\circ}$ & $E, E$ & némuphors & + & $\begin{array}{c}+ \\
\text { pontes } \\
\text { nombr. }\end{array}$ & + & + & + \\
\hline $\begin{array}{l}\text { XI Kéliba } \\
15 \text { bon rolda }\end{array}$ & marieot & pierteux & $\begin{array}{l}\text { rémupinars } \\
\text { carex }\end{array}$ & & & néruphars & $+H_{i}$ & + & & & \\
\hline
\end{tabular}

\footnotetext{
Biom。 = Biomphalaria pfeifferi gaidi

Bul. = Bulinus guernei

Anis. = Anisus spo
}

Nous mentionnons seulement pour mémoire "existence de très nombreux gros mollusques tout le long des bords du fleuve Casamance, mais qui ne jouent aucun rôle dans

\section{iyno = Lyrinaea caillaudi}

э. s. = Bulinus senegalensis

l'épídémiologie des affections à trématodes de l'homme et des onimaux domestiques). 
TABLEAU II - Infestation des mollusques.

\begin{tabular}{|c|c|c|c|c|c|c|}
\hline \multirow{2}{*}{$\begin{array}{c}\text { Mollusques } \\
\text { Espèce }\end{array}$} & \multicolumn{6}{|c|}{ Formes larvaires trouvées à la dissection } \\
\hline & $\begin{array}{r}\text { Fasciola } \\
\text { gigantica }\end{array}$ & $\begin{array}{l}\text { Sch. hae- } \\
\text { matobium }\end{array}$ & $\begin{array}{l}\text { Xiphido- } \\
\text { corcaires }\end{array}$ & $\begin{array}{c}\text { Cercaires } \\
\text { cysto- } \\
\text { phores }\end{array}$ & $\begin{array}{c}\text { Echinosto- } \\
\text { midae }\end{array}$ & $\begin{array}{l}\text { Amphisto- } \\
\text { midae }\end{array}$ \\
\hline $\begin{array}{l}\text { Lymnaea } \\
\text { caillaudi }\end{array}$ & 60 à $80 \%$ & & $10 \%$ & & & \\
\hline $\begin{array}{c}\text { Biomphalaria } \\
\text { pfeifferi gaudi }\end{array}$ & & & $10 \%$ & $10 \%$ & $5 \%$ & \\
\hline $\begin{array}{l}\text { Bulinus } \\
\text { Euernei }\end{array}$ & & 85 à $90 \%$ & & & & \\
\hline $\begin{array}{l}\text { Bulinus } \\
\text { senegalensis }\end{array}$ & & & & & & $15 \%$ \\
\hline $\begin{array}{l}\text { Anisus } \\
\mathrm{sp} \text {. }\end{array}$ & & & & & $15 \%$ & \\
\hline
\end{tabular}

Tous ces animaux provenaient de la région, et sur 24 examinés, 14 d'entre eux étaient parasités par Fasciola gigantica: 7 massivement, 6 avaient une forte infestation, 1 seulement ne présentait que quelques douves dans ses canaux biliaires.

Le registre des saisies de cet abattoir signale pour un abattage quotidien de 3 à 4 bovidés, un ou deux foies saisis pour distomatose. Comme le centre de Kolda se ravitaille en viande dans les villages des environs sans avoir recours à l'importation d'animaux d'autres régions, c'est pratiquement 30 à 50 p. 100 des bovins adultes aui sont atteints de distomatose dans cette partie de la Haute-Casamance.

\section{HÔTE INTERMÉDIAIRE DE FASCIOLA GIGANTICA AU SÉNÉGAL}

D'après les récoltes que nous avons pu faire dans les points d'eau de différentes régions, la Limnée que l'on trouve au Sénégal appartient à l'espèce Lymnaea caillaudi (*).

(*) A ce sujet nous remercions monsieur le professeur G. RANSON du Muséum d'Histoire Naturelle de Paris, qui a bien voulu nous déterminer le matériel malacologique que nous avons récolté.
Pour contrôler la valeur de ce mollusque comme vecteur hôte intermédiaire de $F$. gigantica au Sénégal, nous avons procédé, au laboratoire, à des infestations expérimentales sur des $L$. caillaudi d'élevage, une souche de Kolda (Casamance), et une souche de Thiès-Fandène (Cayor).

\section{Protocole expérimental.}

La récolte d'cufs de $F$. gigantica dans des fèces de bovidés est longue et fastidieuse. Par cette méthode on introduit d'autre part dans les incubateurs de nombreux protistes, (en porticulier des Ciliés), qui entravent le bon développement des embryons de douves. C'est pourquoi nous avons préféré prélever des oufs dans la vésicule biliaire des animaux atteints de distomatose.

Pour se procurer des milliers d'œufs de F. gigantica it suffit de récupérer le contenu de la vésicule biliaire d'un foie saisi aux abattoirs pour fasciolose.

Ces pontes sont lavées une dizaine de fois pour les débarrasser de la bile, puis mises à incuber dans de l'eau de gîte à mollusques filtrée. Les incubateurs sont maintenus pendant environ un mois à une température de $25^{\circ}$ $26^{\circ} \mathrm{C}$.

Des contrôles réguliers, faits tous les jours à la période ultime de l'incubation, permettent de 
repérer, à quelques heures près, la date de sortie des miracidia.

Pour l'infestation de l'hôte intermédiaire, les miracidia sont mis en présence des Limnées pendant 2 heures à la température de 290. $30^{\circ} \mathrm{C}$; on a soin de limiter leur nombre en fonction de celui des mollusques à infester, si l'on veut éviter une trop forte mortalité des Limnées dans les jours qui suivent l'expérience.

A partir de ce jour, des dissections régulières sont faites dans les lots de Limnées pour suivre le rythme d'apparition, l'évolution des formes larvaires et l'époque où apparaissent les cercaires mûres prêtes à sortir du mollusque.

\section{Résultats de l'expérimentation.}

Au cours de deux essais, I'un fait sur 50 , l'autre sur 130 L. caillaudi, nous avons pu faire les remarques suivantes:

A $25^{\circ}-26^{\circ} \mathrm{C}$, les pontes de F. gigantica donnent des miracidia en 26 à 30 jours.

Un seul miracidium est capable d'infester une Limnée, et les deux souches: Kolda ei Thiès-Fandène sont également sensibles.

Les premières rédies apparaissent dès le 3 e ou 4 jour.

Au bout de trois semaines d'évolution. le nombre des rédies est déjà considérable dans l'hépato-pancréas du mollusque.

Dès le 25 e jour on peut trouver des cercaires différenciées chez quelques rédies.

En travaillant à $25^{\circ}-26^{\circ}$ les premières cercaires sortent des mollusques entre le $35^{\mathrm{e}}$ et le $40^{\mathrm{e}}$ jour. En maintenant les Limnées à 200-230, elles n'émettent des cercaires que vers le $50^{\circ}$ ou $60^{\circ}$ jour.

\section{ÉPIDÉMIOLOGIE DE LA DISTOMATOSE BOVINE EN RÉGION DE KOLDA}

Les résultats trouvés à la dissection des Lymnées récoltées à Kolda, et dans ses environs, sont en corrélation avec ceux trouvés à l'examen des viscères des bovins à l'abattoir de ce centre.

La grande dispersion et le haut pourcentage d'infestation de ces mollusques par des formes larvaires de la douve géante, suffisent à expliquer la fréquence de la distomatose dans une contrée où les cours d'eau (Casamance et marigots affluents) ont des crues longues et importantes au cours de l'hivernage.

En nous basant sur quelques observations faites pendant notre séjour à Kolda, où nous avons pu assister à deux crues de la Casamance ayant fait monter le niveau de l'eau de plusieurs mètres, rous supposons 'qu'au point de vue épidémiologique, la fréquençe de cefte affection parasitaire puisse s'expliquer de la manière suivante:

L'infestation des $L$. coilloudi doit surtout se produire au moment des basses eaux, durant la saison sèche, quand les bovins vont boire à la Casamance ou aux marigots permanents.

Une forte densité de mollusques, dans une petite quantité d'eau, avec un courant presque nul, sont autant de facteurs pouvant favoriser l'infestation des Limnées par les nombreux miracidia. sortant des œufs de douves évacués avec les excréments des arimaux parasités.

Par contre, en période de crue, le milieu aquatique ne présente pas les conditions optima pour que l'évolution ouf-miracidium se fasse dans de bonnes conditions. Le courant très fort disperse les cufs, et les miracidia qui éclosent sont emportés par la crue. Les gîtes à hôtes intermédiaires sont perturbés ef les conditions de repos nécessaires pour une pénétration normale du miracidium chez le mollusque ne sont plus réalisées.

Au point de vue dissémination des métacercaires, pendant l'hivernage, les grandes pluies provoquent de très forles crues qui emportent à la dérive un grand nombre de mollusques tout au long de la Casamance et de ses affluents.

Nous avons pu constater qu'à la suite d'une brusque montée des equx :

10 Les gîtes à mollusques repérés. la veille de la crue avaient été détruits.

$2^{\circ}$ Lc densité malacologique par mètre carré avait considérablement diminué.

30 Les mollusques étaient emportés à la dérive fixés à des fevilles et des racines de Pistia L., ou d'Aeschornia, ou à des fragments de bois pourri. 
40 Dcux jours après la crue alors que les eaux étaient descendues d'environ 1 mètre, la densité malacologique avait sensiblement augmenté. II était alors possible de trouver quelques mollusques fixés soit sur les graminées du bord, soit sur des feuilles de Nymphaea Smith, au milieu du fleuve.

Tout ceci laisse supposer que, dès que la force et la vitesse du courant diminuent, les mollusques se réfugient dans les endroits où l'eau est plus tranquille, principalement le long des rives du fleuve. Là, les conditions de chaleur et d'insolation de l'eau étant favorables, les spécimens infestés émettent des cercaires qui vont s'enkyster sur les végétaux immergés par l'inondation.

En fin d'hivernage, quand les eaux onl baissé et que la Casamance et les marigots affluents sont réduits à des filets d'eau ou à des mares, le bétail s'infeste en avalant les métacercaires fixées sur les herbes des rives qui ne sont plus submergées.

En ce qui concerne l'écologie et la conservation des espèces de mollusques dans la Casamance au cours de l'hivernage, nous avons remarqué que la dispersion des adultes est très largement compensée par les nombreuses pontes fixées à la face inférieure des fevilles de nénuphars, très abondants dans le lit de la Casamance. Ces feuilles représentent un support excellent puisqu'elles sont toujours en surface et qu'elles résistent au courant. En effet, submergées par la première crue, elles émergent au bout de quelques jours. Par un mécanisme d'auto-défense, les tiges qui les relient aux racines fixées au fond du fleuve poussent démesurément $(3,4$ et 5 mètres au niveau du pont do Kolda), jusqu'à ce qu'elles arrivent à la surface de l'eau.

Les Nymphaea paraissent donc jover un rôle important dans l'écologie des mollusques hôtes intermédiaires, et par voie de conséquence, dans la forte endémicité distomienne existant dans cette région de Haute Casamance.

\section{Remarques :}

Les marigols affluenls de la Casamance, qui tarissent au cours de la saison sèche, sont envahis par les $L$. caillaudi à l'occasion des crues.

C'est la Casamance qui joue le rôle de gîte permanent à Limnées en période de basses eaux, en assurant leur survie. L. caillaudi en effet, ne résiste pas à une dessiccation prolongée.

Les marigots, dont les lits sont très peu encaissés, contribuent à la dispersion des métacercaires de F. gigantica dans tous les pacages de la région ef ne sonl que des gîtes temporaires.

\section{OBSERVATIONS SUR LA RÉPARTITION GÉOGRAPHIQUE DE L. CAILLAUDI ET SUR CELLE DE LA DISTOMATOSE BOVINE AU SÉNÉGAL}

Au cours de quelques prospections malacologiques que nous avons pu faire dans différentes régions du Sénégal nous avons obtenu les résultats suivants :

a) Région de Velingara $(100 \mathrm{~km}$ à l'est de Kolda).

L. caillaudi est absent dans les ruisseaux et marigots des environs de ce centre, malgré une très forte densité de Biomphalaria pfeifferi gaudi et Bulinus guernei (Grétillat, 1960 a).

b) Région de Basse Casamance (Oussouye $=$ sud-ouest de Ziguinchor).

Absence totale de faune malacologique dans les points d'eau pourtant nombreux de cette région. Les terrains sont pendant une grande partie de l'année envahis par des eaux plus ou moins saumâtres; cette salinité doit rendre le milieu incolonisable par les gastéropodes d'eau douce (Grétillat, 1960 o).

c) Région du Sine-Saloum (Kaffrine, Koungheul, Birkelane).

Alors que Bulinus senegalensis est trouvé en très grand nombre dans les mares non permanentes et les marigots de la région, on ne trouve des $L$. callaudi que dans les gros ruisseaux affluents de la Gambie. Ces limnées sont d'ailleurs infestés par des formes larvaires de F. gigantica, et il existe des foyers de distomatose bovine dans les villages proches de la frontière gambienne $\left({ }^{*}\right)$.

(*) Renseignements recueillis auprès des agents du Service de l'Elevage de Birkelane, Keffrine et Koungheul. 
d) Région du Sénégal oriental (TambacoundaOuest : Malemé-Niani, Koussanar, Koumpemtoum) (Grétillat, 1960 b et 1961).

\section{e) Région de Thiès (Cayor).}

Beaucoup de marigots permanents sont des gîtes de L. caillaudi, B. pfeifferi gaudi, B. guernel et $B$. senegalensis. Nous avons disséqué un grand nombre de $L$. caillaudi provenant d'un marigot situé à $8 \mathrm{~km}$ de Thiès (Fandène). Sur environ 300 exemplaires examinés, nous n'en avons trouvé aucun porteur de formes larvaires de F. gigantica, ef cela malgré la présence de très nombreux troupeaux de bovins venant boire à ce point d'eau. Ces contrôles ont été faits à deux époques différentes: mars 1960 et juillet 1960, l'un en saison sèche, l'autre au début de la saison des pluies.

\section{f) Région du Cap-Vert.}

L. caillaudi est nombreux dans les marigots ef les mares permanentes des environs de Dakar, et notamment à Sangalkam, où il voisine avec $B$. pfeifferi gaudi et $B$. senegolensis. LARIVIĖRE et CHARNIER, en 1957, ont fait les mêmes constatations.

De toutes les Limnées disséquées, nous n'en avons trouvé aucune infestée par des formes larvaires de la douve géante, alors qu'un très fort pourcentage l'était par des xiphidocercaires.

\section{Discussion.}

Le fait de ne pas trouver de L. caillaudi infestées pur $F$. gigantica dans ces deux dernières régions, nous a fait penser tout d'abord d̀ l'existence d'une «race » ou plutôt d'une souche ce Limnées réfractaire à l'infestation par des nirccidia de douve géante. Mais, par la suite, nous avons réussi à infester expérimentalement des L. caillaudi provenant de Thiès-Fandène, et cette hypothèse n'a pas de valeur.

L'autopsie d'animaux ayant vécu pendant plusieurs années à la Ferme de Sangalkam. (Ferme du Laboraluire national de recherches vétérinaires de Dakar), montre l'aosence de douves ou seulement la présence de cuelques parasites dans les canaux biliaires de ces bovins. (Cas d'une vieille vache n'ayant pas quitté la ferme depuis dix ans, et à l'autopsie de laquelle on ne récolta que trois douves (avril 1961).

Devant un tel état de chose nous pensons que pour ces deux régions, il s'agit d'une rupture du cycle biologique du parasite au niveau de l'infestation du ruminant par la métacercaire.

En effet, dans cette partie du Sénégal, de par la nature du terrain et le régime des pluies, les gîtes à mollusques, qui sont en général des marigots s'asséchant partiellement en saison sèche, ont des crues très brutales, avec une décrue très rapide ramenant le cours d'eau dans son lit quji est en général encaissé.

L'émission des cercaires, ne se produisant que dans une eau relativement calme, ne peut avoir lieu que lorsque le cours d'eau est rentré dans son lit. Ces cercaires ne peuvent donc s'enkyster que sur des végétaux poussant dans le fond du ruisseau ou du marigot. La distribution des métacercaires fixées sur des plantes qui ne sont pas broutées par le bétail romprait le cycle biologique du parasite.

Tout ceci n'a que la valeur d'une hypothèse, mais permettrait d'expliquer la rareté de la distomatose bovine dans des régions où existe en abondance son mollusque hôte intermédicire.

\section{CONCLUSION}

Comme au Kenya où DINNIK et DINNIK en 1956. ont étudié le cycle évolutif de Fasciola gigan$+1 c 0$, c'est Lymnaea caillaudi Bourguignat, qui est cu Sénégal l'hôte intermédiaire de ce trématode.

Au sujet de la durée de l'évolution des formes larvaires chez le mollusque, les résultats que nous avons trouvés au laboratoire concordent avec ceux donnés par ces auteurs et par ALICATA en 1938 aux lles Hawai chez Fossaria Olluio.

Lc répartition géographique du parasite ne se superpose pas exactement au Sénégal avec celle du mollusque vecteur.

Alors cu'en Haute-Casamance (Kolda) règne une haute endémicité distomienne, la presqu'île du Cap Vert et le Cayor (Thiès) semblent être exempts de distomatose bovine malgré la présence de irès nombreux $L$. callaudi dans les points d'eau. 
D'autres parties du Sénégal restent à prospecter, en particulier la vallée du fleuve Sénégal et le lac de Guiers où la fasciolose bovine existe à l'état endémique.

Au point de vue pratique, l'existence en HauteCasamance d'une très forte endémicité bilharzienne à Schistosoma haematobium Bilharz, 1858, associée à la fréquence de la distomatose bovine. devrait permettre la mise sur pied d'un programme de lutte anti-mollusques par intervention concertée et conjuguée des Services de
Santé et de l'Elevage. En effet, I'hôte: intermédiaire de Sch. haematobium, qui est au Sénégal Bulinus guernei, voisine dans le fleuve Casamance avec Lymnaea cailloudi, et une même intervention molluscocide pourrait détruire ces deux mollusques vecteurs.

\section{Institut d'élevage ef de médecine vétérinoire des pays tropicaux : Laboratoire natio- nal de recherches vétérinaires « Georges Curasson 》, Dakar-Hann (Sénégal).}

\section{SUMMARY}

\section{Preliminary Note on Bovine Distomatosis in Senegal.}

Bovine distomatosis is of high incidence in Senegal. In some regions 30-50\% of the animals slaughtered are infested with Fasciola gigantica (Cobbold, 1885). A survey of the snail factor in the epidemiology of the disease and experimental infestations have shown that the mollusc vector is Lymnea caillaudi Bourguignat.

The author has examined and discusses the factors in the life cycle of this trematode, in the different regions of Senegal, which influence infestation.

\section{RESUMEN}

\section{Nota preliminar sobre la epidemiología de la distomatosis bovina en el Senega!.}

La distomatosís bovina es frecuente en el Senegal. En ciertas regiones, se encuentra de un 30 a 50 p. 100 de los animales sacrificados. El agente de esta afección es Fasciola gigantica Cobbold, 1885. Investigaciones epidemiológicas han mostrado que el molusco vector es Lymnea caillaudi Bourguignat. El autor examina y discute los factores que influyen, en diferentes regiones del Senegal, sobre el ciclo del parásito.

\section{BIBLIOGRAPHIE}

ALICATA J. A. (1938). - Observations on the life history of Fasciola gigantica, the common liver fluke of cattle in Hawaii, and the intermediate host, Fossaria ollula. Hawaii Agric. exper. Station. Bull., 80 : 1-22.

DINNIK J. A, and DINNIK N. N. (1956). Observations on the Succession of Redial
Generations of Fasciola gigantica Cobbold in a Snail Host. Zsch. Tropenmed. Parasito., 7(4) : 397-419.

GRETILLAT S. (1960 a). - Rapport d'enquêtes parasitologiques faites en Casamance. Laboratoire central de l'Elevage « Georges Curasson » Dakar. Août 1960 (non publié). 
GRETILLAT S. (1960 b). - Rapport sur les résultats d'une enquête malacologique effecfuée dans la région de Tambacounda (Sénégal Oriental). Laboratoire central de l'Elevage « Georges Curasson » Dakar. Décembre 1960 (non publié).

GRETILLAT 5. (1961). - Epidémiologie de la bilharziose vésicale au Sénégal Oriental. Observations sur l'écologie de deux bulins:
Bulinus guernei el Bulinus senegalensis. Bull. Org. mond. Santé (sous presse).

LARIVIÉRE M. et CHARNIER M. (1957). - Contribution à l'étude des bilharzioses au Sénégal. Recherche des mollusques sur la presqu'île du Cap-Vert. Bull. Mém. Ecole notion. Méd. Pharm. Dakar, 5 : 336-9.

MOREL P. C. (1959). - Les helminthes des animaux domestiques de l'Afrique occidentale. Rev. Elev. Méd. vét. Pays trop., 12 (2) : 153-174. 